\title{
Reconstruction of Tokamak Equilibrium from Measured Magnetic Data with Error Radial Field
}

\author{
Mitsushi Abe and Kazuhiro Takeuchi \\ Energy Research Laboratory, Hitachi Ltd., Hitachi 316 \\ (Received April 8, 1991/Revised Manuscript Received December 27, 1991)
}

\begin{abstract}
A tokamak plasma equilibrium code to reconstruct the magnetic field around a plasma from measured magnetic data, was applied to the Hitachi tokamak (HT-2). It was found that a systematic measurement error is produced by the toroidal field coils and it makes an iterative calculation converge to a wrong equilibrium state. A technique to compensate for the measurement error field was developed. The plasma cross-sectional shape is then well analyzed in the HT-2 experiments. A compensating radial field of around $5 \times 10^{-4} \mathrm{~T}$ is necessary for a successful equilibrium calculation at a toroidal field of $1 \mathrm{~T}$. This value of the compensating field is consistent with the results of the experiments to optimize breakdown. This technique is applicable to all tokamaks.

Keywords:

tokamak equilibrium, poloidal field, magnetic analysis, magnetic measurement, error field,
\end{abstract}

\section{Introduction}

The determination of a plasma cross-sectional shape through an analysis of magnetic data is basic and important problem in tokamak experiments ${ }^{1-3)}$. Several codes to carry out the magnetic analysis using experimentally obtained magnetic data have been developed and used in non-circular tokamak plasma experiments ${ }^{4-7}$. In the Hitachi Tokamak (HT-2) experiments, plasma cross-sectional shapes are usually determined by the SHP code ${ }^{8,9)}$. A plasma current in modelled by a few filamental loop currents and the loop currents as well as coil currents are determined so as to reconstruct the measured magnetic field. The SHP code similar to the codes of refs. 4) and 6) features a short computational time and a reasonable accuracy. However it differs from the codes of refs. 4) and 6) in taking the eddy currents on a vacuum vessel wall into account. The eddy currents are not negligible in HT-2 because of a small plasma current $\left(I_{\mathrm{p}}=10-55 \mathrm{kA}\right)$ and a low vessel loop resistance $(14 \mathrm{~m} \Omega)$. Reference 7$)$ has pointed out that the accuracy of codes like SHP is degraded in highly elongated plasma experiments. To avoid it, a full MHD calculation code with an efficient technique has been developed for the Doublet III experiments ${ }^{7)}$.

Applying the algorithm of ref. 7) to $\mathrm{HT}-2$, we have found a problem. A systematic measurement error which couples with an instability makes an iterative calculation diverge or converge to a wrong equilibrium state. A typical example is that the systematic measurement error of a radial (or horizontal) field couples with the vertical position instability of an elongated plasma. The cause of the systematic measurement error is found to be that the poloidal component (error field component) of the magnetic field produced by the toroidal 
field coils can not be measured generally. Therefore we have developed a tokamak equilibrium code FITEQ, which compensates for the radial field component of the systematic measurement error during the iterative calculation. The name FITEQ stands for a field input type equilibrium code.

The objectives of this paper are to show the effect of the systematic measurement error of the radial field on the magnetic analysis and to present techniques to compensate for it in the magnetic analysis using FITEQ code.

\section{Method to obtain equilibrium magnetic field from measured data}

\section{1 Systematic measurement error of radial field}

The experimental data have statistically random measurement errors and systematic measurement errors. When a large number of data are taken into account, the former errors are averaged and can be ignored. However, the latter errors are not averaged and can not be ignored. The magnetic field data obtained through a tokamak experiment have a systematic error described below.

The force balance on a plasma is one of important informations in the magnetic analysis. The vertical force $F_{z}$ acting on the plasma should be zero when the plasma is in an equilibrium state. We have used a magnetic analysis code called SHP ${ }^{8,9)}$, which models a plasma current by a few filamental loop currents but can not analyse the magnetic field in the plasma area. We have found that the analysed value of $F_{z}$ is always a non zero value. This means that there is a systematic measurement error of radial field $B_{\mathrm{R}}{ }^{\mathrm{E}}$ in the plasma area. In the analysis of magnetic data, the plasma is experiencing the error radial field of a magnitude given by

$$
\begin{aligned}
& B_{\mathrm{R}}^{\mathrm{E}}=-F_{\mathrm{z}} /\left(2 \pi R_{\mathrm{p}} I_{\mathrm{p}}\right), \\
& F_{z}=\frac{1}{2 \mu_{0}} \int 2 \pi R B_{\mathrm{p}}^{2} \boldsymbol{n} \boldsymbol{e}_{z} \mathrm{~d} l,
\end{aligned}
$$

where the integration is taken along the plasma surface contour on a poloidal cross-section, $\boldsymbol{n}$ is a normal vector to the plasma surface, $\boldsymbol{e}_{z}$ is a unit vector along the vertical axis, $B_{\mathrm{p}}$ is the poloidal field on the plasma surface, $R_{\mathrm{P}}$ and $I_{\mathrm{p}}$ are the plasma major radius and a plasma current, respectively. To estimate the source of this measurement error field $B_{\mathrm{R}}{ }^{\mathrm{E}}$, the dependence of $B_{\mathrm{R}}{ }^{\mathrm{E}}$ on the toroidal field $B_{\mathrm{T}}$ and plasma current $I_{\mathrm{p}}$ was examined. The results are shown in Fig. 1 , in which $B_{\mathrm{R}}{ }^{\mathrm{E}}$ is plotted as a function of $B_{\mathrm{T}}$ for two groups of $I_{\mathrm{p}}$. The magnitude of $B_{\mathrm{R}}{ }^{\mathrm{E}}$ is proportional to $B_{\mathrm{T}}$ but has no clear dependence on $I_{\mathrm{p}}$, which means that $B_{\mathrm{R}}{ }^{\mathrm{E}}$ is directly related with $B_{\mathrm{T}}$.

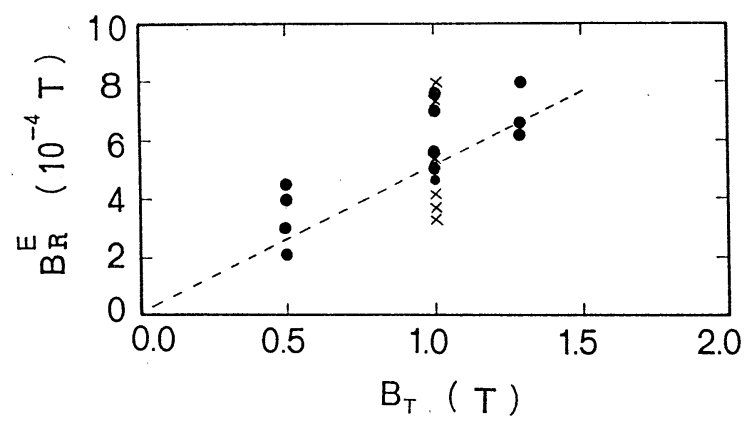

Fig. 1 Correlation between systematic measurement error in radial field $B_{\mathrm{R}}{ }^{\mathrm{E}}$ and toroidal field $B_{\mathrm{T}}$. Dots are the data obtained with plasma current $I_{\mathrm{p}}=12-19 \mathrm{kA}$ and crosses with $I_{\mathrm{p}}=33-39 \mathrm{kA}$. 
A possible source of this measurement error is thought to be an error field component of the magnetic field produced by toroidal field coils. The error field cannot be ignored as in JFT-2 ${ }^{10)}$ generally. The reason why the error field can be a source of the systematic measurement error $B_{\mathrm{R}}{ }^{\mathrm{E}}$ is that such error field component is not measured by the diagnostic system. The error field due to the toroidal field coils has components other than the radial component, generally. When $\psi^{\mathrm{T}}$ and $\psi$ denote error field and real field flux functions, respectively, the poloidal flux function $\psi^{\mathrm{m}}$ measured by a usual magnetic diagnostic system is

$$
\psi^{\mathrm{m}}=\psi-\psi^{\mathrm{T}} .
$$

The measured flux function $\psi^{\mathrm{m}}$ is equal to $\psi$ subtracted by $\psi^{\mathrm{T}}$ but not to $\psi$.

The reason why $\psi^{\mathrm{T}}$ is not measured is as follows. The integration of magnetic sensor signals starts after the toroidal field is applied in tokamak experiments. This situation is shown in Fig. 2 , in which schematic waveforms of $B_{\mathrm{T}}, I_{\mathrm{p}}$, the radial field $B_{\mathrm{R}}$ (real radial field including error and all other radial fields) and the measured radial field $B_{\mathrm{R}}{ }^{\mathrm{m}}$ in the plasma area are shown. The signals of $B_{\mathrm{R}}$ and $B_{\mathrm{R}}{ }^{\mathrm{m}}$ correspond to signals of $\psi$ and $\psi^{\mathrm{m}}$, respectively. The radial field $B_{\mathrm{R}}$ is not zero at the beginning of the integration, which starts, as shown by an arrow in Fig. 2, after the toroidal field is applied but before the plasma current and poloidal field coil currents starts. When the measurement system starts, the magnetic diagnostic system has been reset and $B_{\mathrm{R}}{ }^{\mathrm{m}}$ is measured to be zero. At this time the error field $\psi^{\mathrm{T}}$ already exist and the radial field $B_{\mathrm{R}}$ is equal to the error radial field $B_{\mathrm{R}}{ }^{\mathrm{T}}$, which is the radial component associated with $\psi^{\mathrm{T}}$. Therefore, the measured radial field $B_{\mathrm{R}}{ }^{\mathrm{m}}$ does not include $B_{\mathrm{R}}{ }^{\mathrm{T}}$ and differs from $B_{\mathrm{R}}$ by $-B_{\mathrm{R}}{ }^{\mathrm{T}}$ (i.e. $B_{\mathrm{R}}{ }^{\mathrm{m}}=B_{\mathrm{R}}-B_{\mathrm{R}}{ }^{\mathrm{T}}$ ). Since the systematic measurement error $B_{\mathrm{R}}^{\mathrm{E}}$ is defined by $B_{\mathrm{R}}{ }^{\mathrm{m}}=B_{\mathrm{R}}+B_{\mathrm{R}}^{\mathrm{E}}, B_{\mathrm{R}}{ }^{\mathrm{E}}$ is opposite in direction to $B_{\mathrm{R}}^{\mathrm{T}}$ $\left(B_{\mathrm{R}}^{\mathrm{E}}=-B_{\mathrm{R}}^{\mathrm{T}}\right)$.
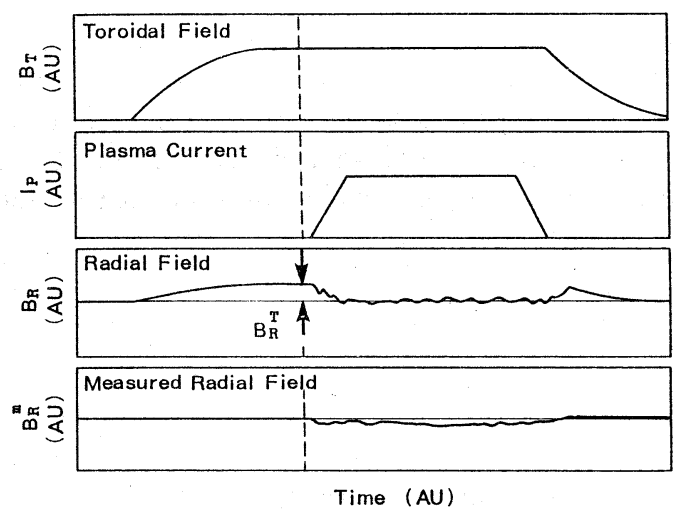

Fig. 2 Schematic waveforms of toroidal field $B_{\mathrm{T}}$, plasma current $I_{\mathrm{p}}$ real radial field $B_{R}$ and measured radial field $B_{R}{ }^{m}$. The magnetic data are measured by integrating the pickup coil signals. The dashed line shows the beginning integration which starts after $B_{\mathrm{T}}$ reaches its steady state value.

On the other hand, the magnetic diagnostic system can start the integration just before the toroidal field coil current starts. In this case the error flux function $\psi^{\mathrm{T}}$ may be measured. However, since it is difficult to distinguish the pick up of the toroidal field due to the setting error of magnetic probes, from the error field due to the toroidal field coils, some systematic measurement error due to $\psi^{\mathrm{T}}$ will still remain in the measured magnetic field data. Then, the magnetic analysis is to be carried out with the systematic measurement error. 


\section{2 Method of reconstructing the tokamak equilibrium magnetic field}

The magnetic field on a poloidal cross-section of a tokamak device including the plasma area is described by an equilibrium equation ${ }^{7)}$,

$$
\begin{aligned}
& L \psi=-\mu_{0} R\left\{j_{\mathrm{p}}(R, \psi)+\Sigma I_{\mathrm{c} i} \delta\left(R-R_{\mathrm{c} i}, Z-Z_{\mathrm{c} i}\right)\right\}, \\
& L=R \frac{\partial}{\partial R} \frac{1}{R} \frac{\partial}{\partial R}+\frac{\partial^{2}}{\partial Z^{2}},
\end{aligned}
$$

where $R, Z, \mu_{0}$ and $j_{\mathrm{p}}$ are radial position, vertical position, permeability in vacuum and plasma current density, and $I_{c i}, R_{c i}$ and $Z_{c i}$ are $i$ th coil current, radial and vertical positions of the coil current, respectively. The problem to be solved is to obtain the distribution of the flux function $\psi(R, Z)$ and the distribution of the plasma current $j_{\mathrm{p}}(R, \psi)$ in the plasma area $(\psi>0)$, which are consistent with the magnetic field measured by the sensors. The flux function $\psi$ is a function of $\boldsymbol{I}_{\mathrm{c}}$ and $j_{\mathrm{p}}$, where $\boldsymbol{I}_{\mathrm{c}}$ is a vector standing for $I_{\mathrm{c} i}$ 's. Magnetic field values corresponding to those measured can be calculated from $I_{\mathrm{c}}$ and $j_{\mathrm{p}}$, which are determined so as to minimize the following residual $E$ :

$$
E=\sum_{j=1}^{m_{\mathrm{p}}}\left\{P_{j}^{\mathrm{m}}-P_{j}^{\mathrm{c}}\left(j_{\mathrm{P}}(R, \psi), \boldsymbol{I}_{\mathrm{c}}\right)\right\}^{2} / \sigma_{j}^{2},
$$

where $P_{j}^{\mathrm{c}}, P_{j}^{\mathrm{m}}, \sigma_{j}$ and $m_{\mathrm{p}}$ are the calculated field values corresponding to the $j$-th magnetic sensor, the measured field value of the $j$-th magnetic sensor, the random measurement error ${ }^{11)}$ of the $j$-th magnetic sensor and the number of magnetic sensors, respectively.

Lao et al. ${ }^{7)}$ proposed a representation of the plasma current density by

$$
j_{\mathrm{p}}(R, \psi)=R \sum_{i=1}^{n} C_{i} I_{\mathrm{p} i} f_{i}^{\mathrm{p}}(\psi)+\sum_{j=n+1}^{n+m} C_{j} I_{\mathrm{p} j} f_{j}^{\mathrm{F}}(\psi) / R,
$$

i. e. the plasma current density is expanded into a few current modes $f_{i}^{\mathrm{p}}$ and $f_{j}^{\mathrm{F}}$, where $I_{\mathrm{p} i}$ and $I_{\mathrm{p} j}$ are total currents for the current modes and $C_{i}$ and $C_{j}$ are normalization factors. This form makes it easy to minimize $\mathrm{E}$. The first and second terms of Eq. (6) represent $R \mathrm{~d} P(\psi) / \mathrm{d} \psi$ and $1 /\left(2 R \mu_{0}\right) \cdot \mathrm{d} F^{2}(\psi) / \mathrm{d} \psi$ where $P$ is the plasma pressure and $F$ is equal to $R B_{\mathrm{T}}$. Usually we take $n=3$ and $m=1$ in the analysis described below and the current modes are,

$$
\begin{aligned}
& f_{1}{ }^{\mathrm{D}}=1-x, \\
& f_{2}{ }^{\mathrm{D}}=x-x^{2}, \\
& f_{3}{ }^{\mathrm{D}}=x^{2}-x^{3}, \\
& f_{4}{ }^{\mathrm{F}}=1-x, \\
& x=\left(\psi_{\text {ax }}-\psi\right) / \psi_{\text {ax }}(\psi=0.0 \text { at plasma surface }),
\end{aligned}
$$

where $\psi_{\text {ax }}$ is the flux function on the magnetic axis. To solve Eq. (4) under the constraint to minimize the residual $\mathrm{E}$ of Eq. (5), iterative calculations are applied as in ref. 7). In the calculations, the toroidal eddy currents $\boldsymbol{I}_{\mathrm{ed}}$ on the vacuum vessel wall are treated in the same manner as in refs. 8) and 9). They are reconstructed from several Fourier components of the poloidal angle and each component is treated as if it were a poloidal coil. The current vector $\boldsymbol{I}=\left\{\boldsymbol{I}_{\mathrm{p}}, \boldsymbol{I}_{\mathrm{c}}, \boldsymbol{I}_{\mathrm{ed}}\right\}$ is fitted to minimize linearly the residual $E$ in each iterative calculation.

\subsection{Technique of compensating the error field}

We have found a problem when the algorithm of section 2.2 is applied to the HT-2. A large error occurs in determination of the plasma cross-sectional shape when a systematic measurement error couples with a plasma position instability. An example is the case of vertically elongated plasma, where the vertical position is unstable against a slight measurement error radial field $B_{\mathrm{R}}{ }^{\mathrm{E}}$. A systematic measurement error of the radial field 
causes a large vertical displacement during the iterative equilibrium calculation. This is not the case in the calculation of SHP code, because it does not carry out the iterative calculation. To avoid this problem a computation code named by FITEQ was developed, which introduces a hypothetical plasma current mode $j^{\mathrm{H}}(Z, \psi)$.

When the technique discussed so far is applied to the experimental data, the equation to treat $\psi^{\mathrm{T}}$ is,

$$
\begin{aligned}
& L\left(\psi^{\mathrm{m}}+\psi^{T}\right)=-\mu_{0} R\left\{j_{\mathrm{p}}\left(R, \psi^{\mathrm{m}}\right)+j_{\mathrm{p}}{ }^{1}\right\}, \\
& j_{\mathrm{p}}{ }^{1}=\frac{\partial j_{\mathrm{p}}(\psi)}{\partial \psi} \psi^{\mathrm{T}} \text { (in the plasma area), }
\end{aligned}
$$

where $\psi^{\mathrm{T}}$ is assumed to be small. The measured magnetic data contain the complete informations about $j_{\mathrm{p}}(R, \psi)$ including $j_{\mathrm{p}}{ }^{1}$ but do not have information about $\psi^{\mathrm{T}}$. The problem is that we have to estimate $\psi^{\mathrm{T}}$ from the informations about $j_{\mathrm{p}}{ }^{1}$ to solve Eq. (8).

In the following discussions, only the error radial field is considered among the fields associated with $\psi^{\mathrm{T}}$, meaning $\psi^{\mathrm{T}}=-R B_{\mathrm{R}}^{\mathrm{T}} Z$, for the following reasons.

(1) The position of a vertically elongated plasma is unstable against a slight radial measurement error field $B_{\mathrm{R}}{ }^{\mathrm{E}}$ in the magnetic analysis and compensation is necessary.

(2) Equation (6) to reconstruct the plasma current distribution has two components differently dependent on the major radius $R$. A measurement error vertical field $B_{\mathrm{V}}{ }^{\mathrm{E}}$ affects on the ratio of the two components but scarecely on the horizontal plasma position. The field $B_{\mathrm{V}}{ }^{\mathrm{E}}$ does not lead to a significant error in poloidal beta $\beta_{\mathrm{p}}$ and normalized internal inductance $l_{i}$. The errors of $\beta_{\mathrm{p}}$ and $l_{i}$ are estimated to be less than 0.05 and can be ignored.

In the following part of this section, we will explain the technique of the FITEQ code. The mode $j^{\mathrm{H}}(Z, \psi)$ is not consistent with Eq. (4) but is expected to represent $j_{\mathrm{p}}{ }^{1}$ of Eq. (8) at the first iterative calculation. Since $\psi^{\mathrm{T}}$ is proportional to $Z$, this mode is expressed as,

$$
j^{\mathrm{H}}(Z, \quad \psi)=C_{5} I_{\mathrm{p} 5}{ }^{\mathrm{H}}(1-x)\left(Z-Z_{0}\right),
$$

where, $I_{\mathrm{p} 5}{ }^{\mathrm{H}}$ is the magnitude of the hypothetical current mode, $Z$ and $Z_{0}$ are the positions of an observation point and of the magnetic axis, respectively and $C_{5}$ is a normalization factor. The position $Z_{0}$ for the $i$-th iterative calculation is obtained in the $(i-1)$-th iterative calculation. This mode should vanish by adding $\psi^{\mathrm{T}}\left(B_{\mathrm{R}}^{\mathrm{T}}\right)$ to $\psi^{\mathrm{m}}\left(B_{\mathrm{R}}^{\mathrm{m}}\right)$. This hypothetical plasma current mode is included as a plasma current mode in Eq. (6). Then Eq. (6) is replaced by,

$$
j_{\mathrm{p}}(R, \psi)=R \sum_{i=1}^{n} C_{i} I_{\mathrm{p} i} f_{i}^{\mathrm{p}}(\psi)+\sum_{j=n+1}^{n+m} C_{j} I_{\mathrm{p} j} f_{j}^{\mathrm{F}}(\psi) / R+C_{5} I_{\mathrm{p} 5}{ }^{\mathrm{H}}(1-x)\left(Z-Z_{0}\right),
$$

in the FITEQ computation. The magnitude $I_{\mathrm{p} 5}{ }^{\mathrm{H}}$ is determined so as to minimize the residual expressed by Eq. (5) in the same manner as the other plasma current modes, and the poloidal field coils at each iterative process. However, $I_{\mathrm{p} 5}{ }^{\mathrm{H}}$ should become zero in compensating iteratively for $\psi^{\mathrm{T}}$. The technique to compensate the error field $\psi^{\mathrm{T}}$ is as follows.

An estimate of the magnitude of $B_{R}{ }^{\mathrm{T}}$ is obtained from the value $I_{\mathrm{p} 5}{ }^{\mathrm{H}}$ because $I_{\mathrm{p} 5}{ }^{\mathrm{H}}$ is roughly proportional to $B_{\mathrm{R}}{ }^{\mathrm{T}}$. The magnitude of the compensating radial field $B_{\mathrm{R}}{ }^{\mathrm{C}}$, which is expected to be equal to $B_{\mathrm{R}}^{\mathrm{T}}$ finally, is estimated in the $i$-th iterative calculation for the $(i+1)$-th compensating field by,

$$
B_{\mathrm{R}}{ }^{\mathrm{C}}{ }_{i+1}=G^{\prime} I_{\mathrm{p} 5}{ }^{\mathrm{H}} i+B_{\mathrm{R} i}{ }^{\mathrm{C}}
$$

where $G^{\prime}$ is a gain to be determined so as to converge the iterative calculation with asymptotically decreasing $I_{\mathrm{p} 5}{ }^{\mathrm{H}}$. The method to supply the compensating radial field $B_{\mathrm{R}}{ }^{\mathrm{C}}$ is rather arbitrary, but it is supplied by the radial field coil ( $\mathrm{DH}-$ coil) of the HT-2 in the FITEQ calculation. A hypothetical current is added to the $\mathrm{DH}$-coil current. Then, Eq. (11) becomes

$$
I_{\mathrm{DH}}{ }^{\mathrm{C}}{ }_{i+1}=G I_{\mathrm{p} 5}{ }^{\mathrm{H}}+I_{\mathrm{DH}}{ }^{\mathrm{C}}{ }_{i} \text {, }
$$


and the $\mathrm{DH}$-coil current $I_{\mathrm{DH}}$ to calculate the reconstructed equilibrium magnetic field is

$$
I_{\mathrm{DH} i}=I_{\mathrm{DH}}{ }_{i}^{\mathrm{f}}+I_{\mathrm{DH}}{ }_{i}^{\mathrm{C}} \text {, }
$$

where the superscripts $f$ and $C$ denote fitted and compensating values, respectively and the subscripts $i$ and $i+1$ denote the values in the $i$-th and $(i+1)$-th iterative calculations, respectively. The magnetic field used for calculating the plasma current distribution function $\{$ Eq. (4) $\}$ in the $(i+1)$-th iterative calculation is obtained using the $\mathrm{DH}$-coil current of Eq. (13). The compensation of the radial error field by Eqs. (11-13) makes the fitted value of $I_{\mathrm{p} 5}{ }^{\mathrm{H}}$ smaller as the iterative calculation proceeds. This is because the compensation adds $B_{\mathrm{R}} \mathrm{C}^{\mathrm{s}}$ to $B_{\mathrm{R}}{ }^{\mathrm{m}}$ and $B_{\mathrm{R}}{ }^{\mathrm{C}}$ becomes equal to $B_{\mathrm{R}}^{\mathrm{T}}$ asymptotically. Finally, the calculated magnetic field represents the equilibrium field.

\section{Application to Experimental Data}

\section{1 Experimental equipment}

The equilibrium code FITEQ was applied to the HT-2 tokamak which is a small tokamak capable of creating various plasma shapes ${ }^{9)}$. The parameters of the HT-2 tokamak are listed in Table I . The poloidal cross-section and the locations of magnetic probes are shown in Fig. 3.

Table I Parameters of HT-2 Tokamak

\begin{tabular}{llll}
\hline Parameters & Symbol & \multicolumn{1}{c}{ Values } & Unit \\
\hline Plasma Current & $I_{\mathrm{p}}$ & $10-55$ & $\mathrm{kA}$ \\
Electron Density (line averaged) & $n_{\mathrm{e}}$ & $1-3 \times 10^{19}$ & $\mathrm{~m}^{-3}$ \\
Toroidal Field & $B_{\mathrm{T}}$ & 1.0 & $\mathrm{~T}$ \\
Vacuum Vessel Loop Resistance & $\Omega_{\mathrm{v}}$ & 14 & $\mathrm{~m} \Omega$ \\
Plasma Major Radius & $R_{\mathrm{p}}$ & $0.39-0.44$ & $\mathrm{~m}$ \\
Plasma Minor Radius & $a_{\mathrm{p}}$ & $0.08-0.12$ & $\mathrm{~m}$ \\
Elongation & $K$ & $0.9-1.45$ & \\
Iron Core Flux Swing & - & \pm 0.062 & $\mathrm{~Wb}$ \\
Shafranov $\wedge\left(=\beta_{\mathrm{p}}+\mathrm{l}_{\mathrm{i}} / 2\right)$ & $\wedge$ & $0.8-1.1$ & - \\
Safety Factor at Surface & $q$ & $>3.0$ & - \\
Discharge Duration & $t_{\mathrm{d}}$ & $<70$ & $\mathrm{~ms}$ \\
\hline
\end{tabular}

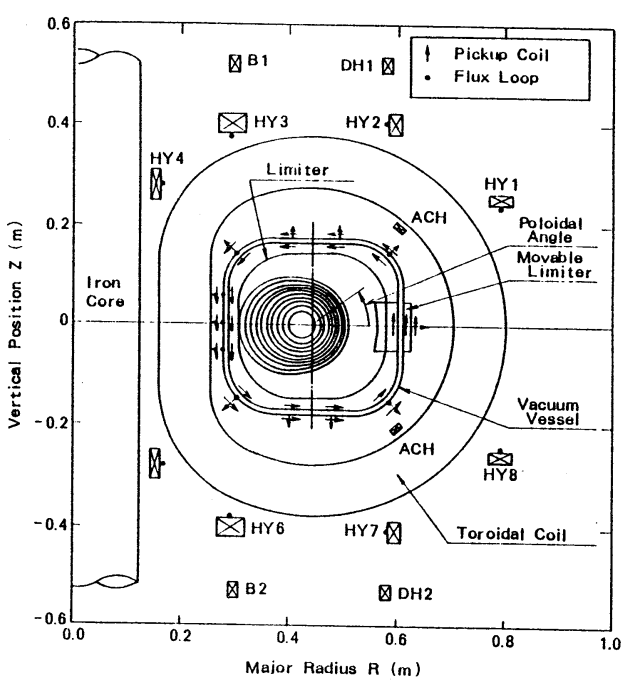

Fig. 3 Poloidal cross-section of the HT-2. Locations of magnetic probes (arrows) and flux loops (small circles) are shown. HY coils supply an arbitrary equilibrium poloidal field, $\mathrm{B}$ coils are for negative biasing of the iron core, $\mathrm{DH}$ coils create a radial field and $\mathrm{ACH}$ coils control the plasma vertical position rapidly. 
The HT-2 has eight HY coils which are not only to maintain the equilibrium poloidal field, but also to drive the plasma current. Each HY coil is connected to one of transistor choppers. The choppers are operated by $1-5 \mathrm{kHz}$ width modulated pulses (PWM). Each HY coil current can be changed independently and various cross-sectional shapes of plasmas are possible. The $\mathrm{ACH}$ coil is operated by a bipolar chopper power supply. An $\mathrm{OH}$ coil can be connected to the chopper power supply, but the plasma current is sufficiently driven by the HY coils for most experiments.

The HT-2 has magnetic sensors of 36 magnetic probes (pickup coils), 15 flux loops and 2 Rogowski coils. A feature of the magnetic sensors is that twelve pairs of magnetic probes and one pair of Rogowski coils are placed so as to make respective pairs inside and outside of the vacuum vessel wall. An additional feature is that each HY coil has a flux loop. The former feature makes it possible to measure the toroidal eddy currents on the vacuum vessel wall. The latter feature solves a problem to reconstruct the magnetic fields due to an iron core and the eddy currents other than on the vacuum vessel wall. They are neither directly measured nor precisely calculated. However, such magnetic fields can be reconstructed in the plasma area by a method in which the measured HY coil currents are not used but the coil currents are fitted so as to reconstruct the measured flux functions at the HY coils. As a result, the coil currents fitted in the equilibrium calculation differ from those really measured in the experiment but have informations about the magnetic field due to the iron core and the eddy currents other than on the vacuum vessel wall. The integration of the pickup signal starts $1 \mathrm{~ms}$ before a breakdown loop voltage is applied in the HT-2 experiment. This situation is the same as in Fig. 2.

\subsection{Example of an equilibrium calculation}

The FITEQ code was applied to plasmas of non-circular cross-sectional shapes of the HT-2. Figure 4 shows the iterative results of the FITEQ calculation. The vertical height of magnetic axis $Z_{\mathrm{p}}$, minimized residual $E$ in each iterative calculation, a convergence parameter and the magnitude of the hypothetical plasma current mode $I_{\mathrm{p} 5}{ }^{\mathrm{H}}$ are shown as functions of the number of iterative calculations. The convergence parameter is expressed by

$$
C_{\mathrm{p}}=\left|\left(\psi^{i}-\psi^{i+1}\right)_{\max } / \psi_{\mathrm{ax}}{ }^{i}\right|,
$$

where the subscript max means that the value is taken at the maximum point in the plasma area and subscripts $i$ and $i+1$ mean that the values are obtained in the $i$-th and $(i+1)$-th iterative calculations. Dots represent the FITEQ calculation with the compensating radial field and crosses that without it. The iterative calculation fails to converge in the actual equilibrium state without the compensation. The residual $E$ increases and $Z_{\mathrm{p}}$ approaches to a wrong value. On the other hand, the iterative calculation reaches fixed values asymptotically with the compensation. The convergence parameter become less than $10^{-3}$ with the compensation, but it cannot be less than about $10^{-2}$ without the compensation. The hypothetical plasma current mode $I_{\mathrm{p} 5}^{\mathrm{H}}$ becomes zero asymptotically as the iterative calculation proceeds. These results show that the calculation technique with $I_{\mathrm{p} 5}{ }^{\mathrm{H}}$ works well and this compensation is necessary for the reconstruction of the equilibrium magnetic field from measured magnetic field data. The compensating radial field $B_{\mathrm{R}}{ }^{\mathrm{C}}$ is $-3.9 \times 10^{-4} \mathrm{~T}$ in the case of Fig. 4 .

Table II shows the measurement error field $B_{\mathrm{R}}{ }^{\mathrm{E}}$ (to be equal to $-B_{\mathrm{R}}{ }^{\mathrm{T}}$ ) obtained from the SHP code using Eq. (1) and the compensating field $B_{\mathrm{R}}{ }^{\mathrm{C}}$ (to be equal to $B_{\mathrm{R}}^{\mathrm{T}}$ and to $-B_{\mathrm{R}}^{\mathrm{E}}$ ) of the FITEQ calculation. The average value of $B_{R}{ }^{C}$ is about $-5 \times 10^{-4} \mathrm{~T}$. The variation of $B_{R}{ }^{C}$ shot-by-shot is due to the uncertainty of measurement. The important point is that absolute values of these $B_{\mathrm{R}}{ }^{\mathrm{E}}$ and $B_{\mathrm{R}}^{\mathrm{T}}$ agree well with each other and $B_{\mathrm{R}}{ }^{\mathrm{E}}$ 's are roughly equal to respective $-B_{R}{ }^{C}$ 's as is expected. The compensating radial field $B_{R}{ }^{C}$ cancels the measurement error radial field $B_{\mathrm{R}}{ }^{\mathrm{E}}$ and adding $B_{\mathrm{R}}{ }^{\mathrm{C}}$ to the magnetic field analysed by the SHP code results in the vanishing of $F_{z}$ of Eq. (1). 


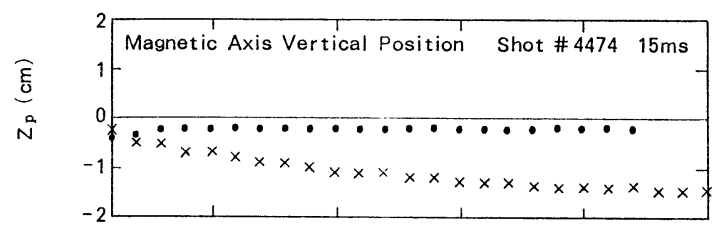

$w$
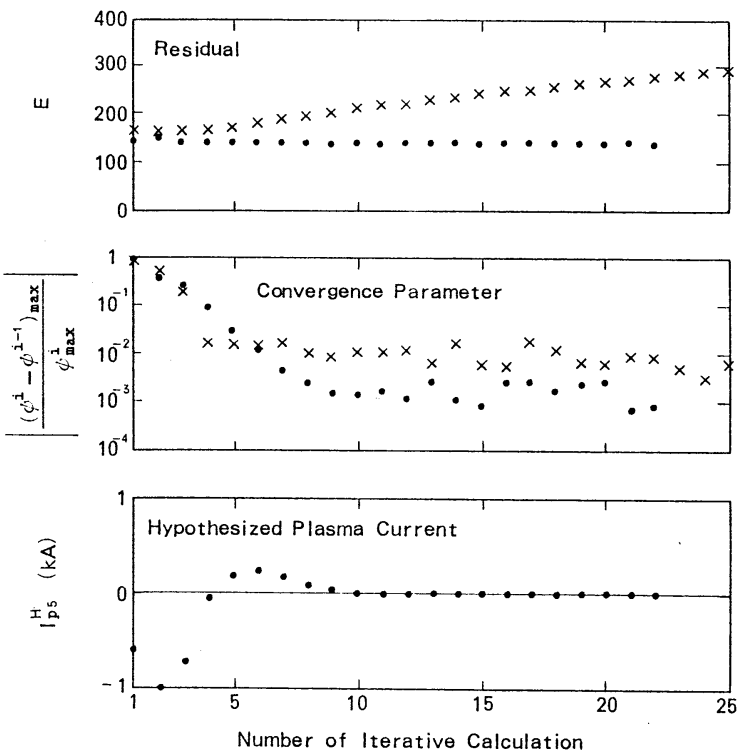

Fig. 4 Iterative results of FITEQ. Magnetic axis height $Z_{p}$, residual $E$, convergence parameter $\left|\left(\psi^{i}-\psi^{i+1}\right)_{\max } / \psi_{\mathrm{ax}}{ }^{i}\right|$ and hypothetical plasma current mode $I_{\mathrm{p} 5}{ }^{\mathrm{H}}$ are shown. Dots represent the calculation with the compensation and crosses that without it.

Table II Estimated measurement error radial field $B_{R}{ }^{E}$ and compensating radial field $B_{R}{ }^{C}$

\begin{tabular}{ccccc}
\hline Shot & $I_{\mathrm{p}}(\mathrm{kA})$ & $F_{\mathrm{z}}(\mathrm{N})$ & $\begin{array}{c}\mathrm{B}_{\mathrm{R}}{ }^{\mathrm{E}}\left(10^{-4} \mathrm{~T}\right) \\
(\mathrm{SHP})\end{array}$ & $\begin{array}{c}\mathrm{B}_{\mathrm{R}}{ }^{\mathrm{C}}\left(10^{-4} \mathrm{~T}\right) \\
(\mathrm{FITEQ})\end{array}$ \\
\hline 4477 & 38.2 & -39 & 3.9 & -3.0 \\
4474 & 37.3 & -80 & 7.9 & -7.9 \\
4471 & 35.5 & -51 & 5.3 & -6.1 \\
4452 & 24.5 & -28 & 4.2 & -3.8 \\
4478 & 38.3 & -33 & 3.3 & -2.6 \\
4460 & 33.7 & -73 & 8.2 & -6.9 \\
\hline
\end{tabular}

Table III Comparison of plasma parameters

\begin{tabular}{llccl}
\hline Parameter & & FITEQ & SHP & Unit \\
\hline Outside Gap & $\delta_{\text {out }}$ & 3.9 & 4.1 & $\mathrm{~cm}$ \\
inside Gap & $\delta_{\text {in }}$ & 1.7 & 1.6 & $\mathrm{~cm}$ \\
Top Gap & $\delta_{\text {top }}$ & 1.4 & 1.7 & $\mathrm{~cm}$ \\
Bottom Gap & $\delta_{\text {bot }}$ & 0.0 & 0.0 & $\mathrm{~cm}$ \\
Plasma Current Center & & & & \\
& $\mathrm{R}_{\mathrm{p}}$ & 43.2 & 43.3 & $\mathrm{~cm}$ \\
& $\mathrm{Z}_{\mathrm{p}}$ & -0.4 & -0.3 & $\mathrm{~cm}$ \\
Minor radius & $\mathrm{a}_{\mathrm{p}}$ & 9.9 & 9.7 & $\mathrm{~cm}$ \\
Elongation & $\kappa$ & 1.41 & 1.39 & \\
$\beta_{\mathrm{p}}+0.5 \mathrm{I}_{\mathrm{i}}$ & $\wedge$ & 1.08 & 1.15 & \\
Internal Inductance & $\mathrm{I}_{\mathrm{i}}$ & 0.89 & - & \\
\hline
\end{tabular}


Figure 5 shows the comparison between the plasma cross-sectional shapes obtained by FITEQ and SHP codes. Since the SHP code does not carry out the iterative calculation, the error field is not a serious problem for the SHP code. Solid lines show the magnetic surface obtained by the FITEQ code and dots show the plasma surface obtained by the SHP code in which the compensating radial field $B_{\mathrm{R}}{ }^{\mathrm{C}}$ is not included. The dots are roughly on the magnetic surface contour touching the limiter at it's bottom, showing that the two obtained plasma cross-sectional shapes agree well with each other. Table III shows the sets of parameters obtained from the two codes. The parameters in respective sets agree roughtly with each other. The gap showing the largest discrepancy is the top gap, which is farthest from the bottom point at which the plasma touches the limiter. The plasma surface is defined by a contour on which the flux function has the same value as on the point at which the plasma surface touches the limiter. Then, the estimated error of flux function $\Delta \psi^{\mathrm{T}}$ associated with the error field $B_{\mathrm{R}}{ }^{\mathrm{T}}$ at the top of the plasma is $\Delta \psi^{\mathrm{T}}=-2 \kappa a_{\mathrm{p}} R_{\mathrm{p}} B_{\mathrm{R}}{ }^{\mathrm{T}}$, where $\kappa$ and $a_{\mathrm{p}}$ are the elongation and the plasma minor radius. Since $B_{\mathrm{R}}^{\mathrm{T}}<0$ and $\Delta \psi^{\mathrm{T}}>0$, the real plasma area should be larger than that obtained by the SHP code in which the calculation of the compensating radial field is not contained. The error of the top gap determined by the SHP code is estimated as,

$$
\Delta \delta_{\mathrm{TOP}^{\mathrm{E}}}^{\mathrm{E}}=2 \kappa a_{\mathrm{p}} B_{\mathrm{R}}^{\mathrm{T}} / B_{\mathrm{p}}
$$

Substituting the parameters of Table III and $B_{\mathrm{p}}=0.04 \mathrm{~T}$ obtained by the SHP code, Eq. (15) yields $0.3 \mathrm{~cm}$ which is equal to the discrepancy between the two top gaps of the Table III , meaning that the discrepancy between the two gaps is mainly due to the error field $B_{R}{ }^{\mathrm{T}}$ and the gaps by the FITEQ code are more accurate than those by the SHP code. Since the error of the gaps $\Delta \delta^{\mathrm{E}}$ are generally proportional to $1 / B_{\mathrm{p}}$, so the error is large at the elongated area (top area of Fig. 5) and the null point area (not null point itself). In this sense, the FITEQ code is more accurate in the non-circular plasma analysis than the SHP code because the error field is successfully compensated.

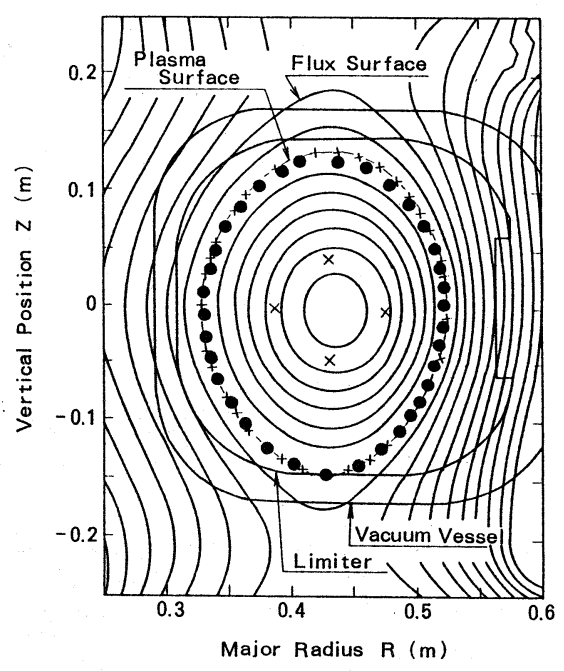

Fig. 5 Magnetic surface reconstructed by the FITEQ code and plasma surface determined by the SHP code. Solid lines show the magnetic surfaces and dots the plasma surface. The four $x$ 's in the plasma area show the locations of filamental currents to model the plasma current distribution in the SHP code. 


\section{3 Breakdown experiment in $\mathrm{HT}-2$}

The compensating radial field $B_{\mathrm{R}}{ }^{\mathrm{C}}$ is examined in breakdown experiments of the HT-2 . Well controlled breakdown is expected to be obtained under the condition of $B_{\mathrm{R}}=0$ in the plasma area, which means that $B_{\mathrm{R}}{ }^{\mathrm{m}}$ is equal to $-B_{\mathrm{R}}{ }^{\mathrm{C}} \fallingdotseq 5 \times 10^{-4} \mathrm{~T}$ (at $B_{\mathrm{T}}=1.0 \mathrm{~T}$ ). The radial field measured at the time of breakdown $B_{\mathrm{R}}{ }^{\mathrm{b}}$ (radial field supplied by the HY-coils at breakdown) can be changed by the HY coil currents. Therefore, breakdown properties are examined by changing $B_{\mathrm{R}}{ }^{\mathrm{b}}$, shot-by-shot. The examined parameters are the delay time $\tau_{\mathrm{d}}$ of breakdown, the peak loop voltage $V_{\mathrm{l}}^{\mathrm{p}}$ and the consumed iron core flux $\Delta \Phi$ during start-up phase $(0-15 \mathrm{~ms})$. Definitions of these parameters are shown in Fig. 6 (a) with the waveforms of the plasma current $I_{\mathrm{p}}$, the loop voltage $V_{1}$ and the consumed iron core flux $\Phi$. The delay time $\tau_{\mathrm{d}}$ is evaluated as the time interval between the starts of the loop voltage and the plasma current. The consumed iron core flux $\Delta \Phi$ is evaluated at the end of the start up phase $(15 \mathrm{~ms})$. The peak loop voltage $V_{\mathrm{l}}^{\mathrm{p}}$ is evaluated at it's peak value. In the experiments the plasma current as well as the plasma positions are well feedback controlled and good reproducibility of the waveforms after the breakdown is obtained. When $B_{R}{ }^{b}$ is not adequate $\left(B_{R}{ }^{b}+B_{R}{ }^{T} \neq 0\right)$, the breakdown is delayed and the HY coil currents are programmed to increase the loop voltage.
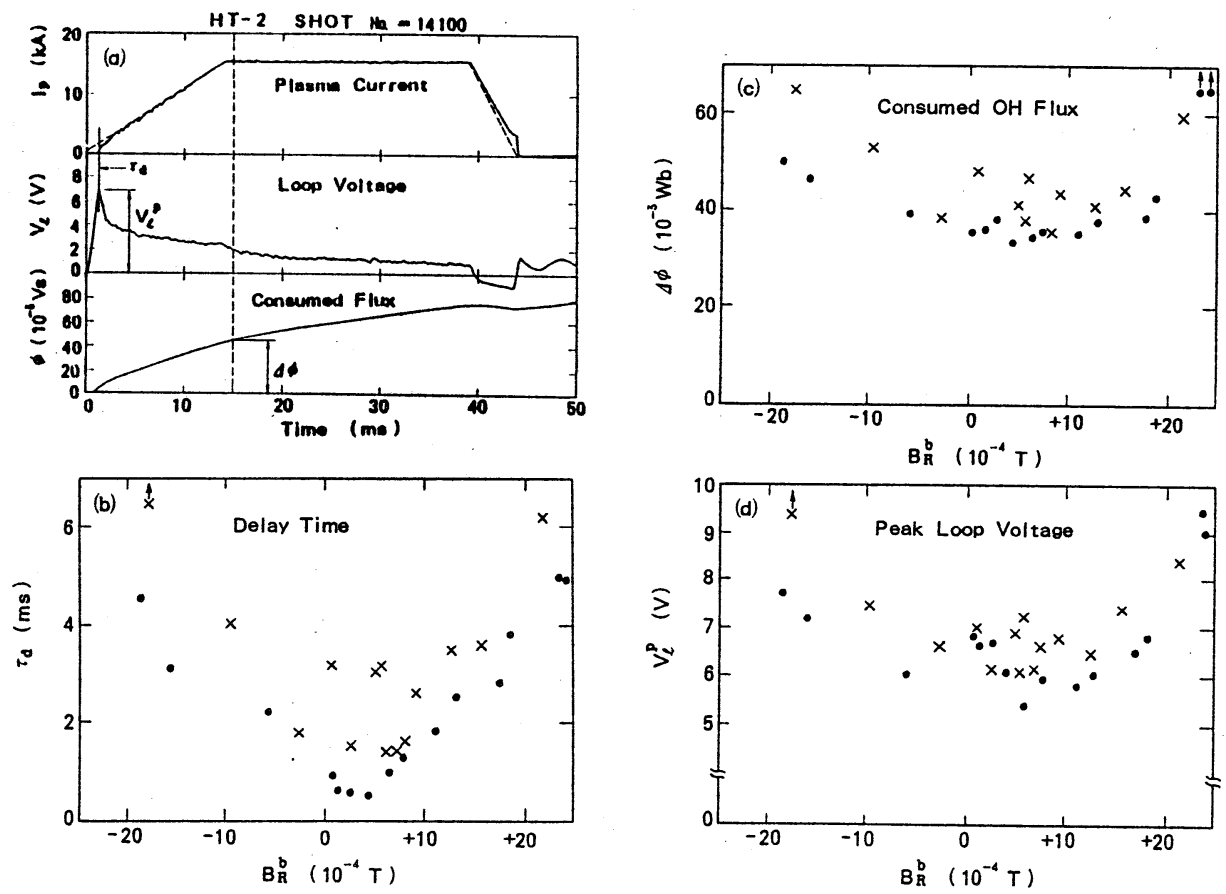

Fig. 6 (a) Time evolution of a discharge in the breakdown experiment. Waveforms of plasma current $I_{\mathrm{p}}$, loop voltage $V_{1}$ and consumed iron core flux $\Phi$ are shown. Dashed line shows a reference signal for the control of $I_{p}$.

(b) Dependence of the delay time $\tau_{d}$ on the applied radial field. Dots show experiments with the preionization and crosses experiments without it. An arrow $\uparrow$ means the breakdown did not occur or the plasma currnet failed to reach $15 \mathrm{kA}$.

(c) Dependence of iron core flux consumption $\Delta \Phi$ on the applied radial field $B_{R}{ }^{b}$.

(d) Dependence of peak loop voltage $V_{\uparrow}^{p}$ on the applied radial field $B_{\mathrm{R}}^{\mathrm{b}}$. 
Figures 6 (b) , (c) and (d) show the values $\tau_{\mathrm{d}}, \Delta \Phi$ and $V_{\mathrm{l}}^{\mathrm{p}}$ as functions of the applied radial field $B_{\mathrm{R}}{ }^{\mathrm{b}}$ at the time of breakdown. Each value is expected to be small under the condition of $B_{\mathrm{R}}=0$ or $B_{\mathrm{R}}{ }^{\mathrm{b}}=-B_{\mathrm{R}}{ }^{\mathrm{C}}\left(\doteqdot 5 \times 10^{-4} \mathrm{~T}\right)$. Figure $6(\mathrm{~b})$ shows the delay time $\tau_{\mathrm{d}}$ as a function of $B_{\mathrm{R}}{ }^{\mathrm{b}}$. The value $B_{\mathrm{R}}$ baries within $\pm 25 \times 10^{-4} \mathrm{~T}$. The breakdown is not obtained with an absolute value of $B_{\mathrm{R}}{ }^{\mathrm{b}}$ greater than $25 \times 10^{-4} \mathrm{~T}$. The results shown by dots are obtained with preionization and those by crosses without it. The preionization is produced by a small electron gun (about $300 \mathrm{~V}, 5 \mathrm{~mA}, 1 \mathrm{~ms}$ ). Without the preionization, the breakdown cannot be obtained at $-18 \times 10^{-4} \mathrm{~T}$, but can be obtained at $22 \times 10^{-4} \mathrm{~T}$, which suggests that there is an error radial field $B_{\mathrm{R}}{ }^{\mathrm{T}}$ which is not measured. In both kinds of experiments (with and without the preionizations), $\tau_{\mathrm{d}}$ is minimized at about $B_{\mathrm{R}}{ }^{\mathrm{b}}=5 \times 10^{-4} \mathrm{~T}$, which is consistent with the value of $B_{\mathrm{R}}{ }^{\mathrm{C}}$ estimated in section 3.2. Figure $6(\mathrm{c})$ shows $\Delta \Phi$ as a function of $B_{\mathrm{R}}{ }^{\mathrm{b}}$. In both kinds of experiments, $\Delta \Phi$ is minimized at around $5 \times 10^{-4} \mathrm{~T}$. Figure $6(\mathrm{~d})$ shows $V_{\mathrm{l}}^{\mathrm{p}}$ as a function of $B_{\mathrm{R}}{ }^{\mathrm{b}}$. The figure has the same tendency as Figs. 6(b) and (c). The lowest breakdown loop voltage $5.4 \mathrm{~V}$ is obtained at $B_{\mathrm{R}}^{\mathrm{b}}=6.4 \times 10^{-4} \mathrm{~T}$, which roughly cancels the error radial field of $B_{\mathrm{R}}{ }^{\mathrm{C}} \fallingdotseq-5 \times 10^{-4} \mathrm{~T}$ estimated by the FITEQ code.

The applied radial field $B_{\mathrm{R}}{ }^{\mathrm{b}}$ to minimize each value of the experiment is rather vaguely determined but each value has its minimum value at $B_{\mathrm{R}}^{\mathrm{b}} \fallingdotseq 5 \times 10^{-4} \mathrm{~T}$. Thus, it can be said that the error radial field $B_{\mathrm{R}}^{\mathrm{T}} \fallingdotseq-5 \times 10^{-4} \mathrm{~T}$ really exists and is not measured by the magnetic sensor system and a well controlled breakdown is obtained when the error radial field is cancelled by the radial field $B_{\mathrm{R}}{ }^{\mathrm{b}}$ applied by the HY coils.

\section{Discussion}

The systematic measurement error should exist in all tokamak devices, because the toroidal field coils generally produce error field components due to the setting error and the feeders. It seems that some compensation as presented in this paper is necessary for an accurate reconstruction of the equilibrium magnetic field. Generally the error field is not as simple as is treated here. Such complex error fields coupled with the instability of the plasma cross $^{-}$sectional shape need a compensating field other than the simple radial field. In the HT-2 experiments, such a necessity has not arisen so far, but might arise in other elongated plasma tokamaks.

It would be better for magnetic analysis codes like SHP (with a few filamental loop currents for a modelled plasma) to compensate for the error field. The top gap error estimated by Eq. (15) agrees with the difference between the results of the two codes (SHP and FITEQ, Table III ). The difference of the plasma conrrent model can be a cause of the difference between the plasma surface positions obtained by the two codes. The plasma current is not a distributed one in the SHP code, while it is distributed in the FITEQ code. However the discussion of Eq. (15) and Table III clarifies that the difference is mainly due to the error radial field $B_{\mathrm{R}}{ }^{\mathrm{T}}$ and not due to the plasma current model used in the analysis.

\section{Conclusion}

A tokamak plasma equilibrium code FITEQ was applied to the Hitachi tokamak (HT-2). The FITEQ code reconstructs the equilibrium magnetic field around the plasma from measured magnetic data. It was found that a systematic measurement error of the radial field is produced by the toroidal field coils and it makes an iterative equilibrium calculation converge to a wrong equilibrium state. A technique to compensate for the error field was developed. The plasma cross-sectional shape is well analyzed in the experiments of the HT-2. An additional radial field of around $-5 \times 10^{-4} \mathrm{~T}$ is necessary for a successful equilibrium calculation at a toroidal field of 1T. The additional field is to compensate for the error field. This value of the compensating radial field is consistent with the results of the breakdown experiments, in which a compensating radial field was applied by the poloidal field coils to optimize the 
breakdown. This technique is applicable to all tokamaks.

\section{Acknowledgements}

We would like to thank Drs. Doi, Ozaki, Oomae, Otsuka, Yoshioka and Murai for their encouragement throughout this work.

\section{REFERENCES}

1) M. Abe, A. Kameari, A. Kitsunezaki, S. Konoshima et al. : Nucl. Fusion 29 (1989) 1659.

2) N. Ohyabu, K. H. Burrell, S. Ejima, R. J. Groebner et al. : Nucl. Fusion 25 (1985) 49.

3) R. M. Wielnd, H. C. Howe, E. A. Lazarus, M. Murakami and C.E. Thomas : Nucl. Fusion 23 (1983) 447.

4) D. W. Swain and G. H. Neilson : Nucl. Fusion 22 (1982) 1015.

5) J.L. Luxon and B. B. Brown : Nucl. Fusion 22 (1982) 813.

6) L. L. Lao, H. St. John, R. D. Stambaugh and W. Pfeiffer: Nucl. Fusion 25 (1985) 1421.

7) L. L. Lao, H. St. John, R. D. Stambaugh, A. G. Kellman and W. Pfeiffer : Nucl. Fusion 25 (1985) 1611.

8) M. Abe, K. Takeuchi, H. Fukumoto and M. Otsuka : Kakuyugo Kenkyu 61 (1989) 38.

9) M. Abe, K. Takeuchi, H. Fukumoto, M. Shimizu and M. Otsuka : Jpn. J. Appl. Phys. 29 (1990) 395.

10) T. Sometani and N. Fujisawa : Plasma Physics 20 (1978) 1101.

11) K. Takeuchi, M. Abe, H. Fukumoto and M. Otsuka: Jpn. J. Appl. Phys. 30 (1991) 398. 